\title{
Les différents types d'erreurs et leur prise en compte dans les calculs géotechniques
}

\section{J.-L. FAVRE}

École centrale de Paris 92295 Châtenay-Malabry
Les trois principales sources d'incertitudes sur les propriétés des sols et des roches sont tout d'abord précisées : la variabilité naturelle, les mesures, les modèles. Leur prise en compte possible est indiquée dans le cadre de l'analyse statistique et de la théorie des probabilités. L'attention est particulièrement attirée sur le problème de la réduction de variance, spécifique aux mécanismes de défaillance sur de grands volumes, et sur le poids très pénalisant du manque de mesures comparativement à la variabilité naturelle des terrains.

Mots clés : variabilité naturelle, incertitudes d'échantillonnage, erreurs de modèles, distance d'autocorrélation, réduction de variance, fiabilité.

\section{The different kinds of errors and their consideration in geotechnical design}

The three main sources of uncertainties about soils and rocks characteristics are described, namely : the natural variability. measures and models. Their possible consideration is described in the statistical analysis and within the theory of probabilities. Attention is specially drawn on the problem of variance reduction, typical in failure mechanisms linked with great volumes, and on the handicap due to the lack of measures.

Key words : natural variability, uncertainties of samplings, model errors, auto correlation distance, variance reduction. reliability. 


\section{1}

\section{Les trois types d'incertitudes pour les sols et les roches}

1.1

\section{Le désordre ordonné}

Le $\propto$ désordre $»$ qui affecte les propriétés des sols et des roches n'est pas aléatoire. Il est lié à l'histoire de leur formation qui a ses lois très précises (tectonique, érosion, transport et sédimentation, etc.) et à la nature même de la matière. Mais la complexité de ces lois et des conditions de leur application et la complexité de la matière font qu'il est impossible de connaitre parfaitement ces propriétés: on est donc devant un phénomène incertain au sens où on ne peut prévoir de façon certaine ses réalisations, i.e. les propriétés en un point d'un massif. La première démarche est de connaître l'essence des phénomènes et pour cela une bonne reconnaissance géologique est toujours indispensable ; elle réduit le caractère incertain de la prévision.

\section{4}

\section{Différents degrés d'incertain en géotechnique}

Cet incertain naturel peut avoir divers degrés et donc entraîner différents moyens d'investigation, de prise en compte et modélisation :

- on peut se trouver dans des terrains très erratiques, à fortes hétérogénéités avec des fractures, des zones de faiblesse ou au contraire des points " durs », des arrivées d'eau, etc. Géométrie et propriétés sont très tourmentées. La modélisation est essentiellement déterministe et dichotomique (oui ou non). Elle peut aussi faire appel aux ensembles flous comme aide à la décision. Une telle démarche est illustrée par l'article de Daniel Boissier " Décision et incomplétude » :

- on peut se trouver dans des terrains peu désordonnés présentant une stratification relativement régulière avec, pour chaque couche, des propriétẻs relativement régulières aussi. La reconnaissance géotechnique, guidée par le modèle géologique de terrain, prend ici toute son importance pour proposer un modèle mécanique de terrain. Les autres articles se placent plutôt dans ce cas où les analyses statistiques et le calcul des probabilités prennent tout leur sens;

- enfin, les terrains peuvent avoir été fabriqués par I'homme, qu'il s'agisse de barrages en terre, de remblais, de terre armée, etc. On a affaire alors à des contrôles de fabrication et des tests de réception. Cet aspect n'est pas traité dans ce numéro spécial.

\section{0}

\section{Différentes échelles de l'incertain}

Un autre aspect de l'incertain naturel des sols et des roches est qu'il peut se manifester à diverses échelles spatiales, emboîtées, fractales. Ceci est illustré par une étude de l'École des mines de Paris sur le massif des Ardennes à propos des propriétés des roches (Fig. 1) : celles-ci décroissent en fonction de l'échelle d'observation ou du volume concerné, depuis l'échelle atomique, où la première dislocation introduit une baisse brusque des propriétés; les échelles suivantes provoquent la poursuite du phénomène tout en l'amortissant de plus en plus jusqu'à l'apparition, à l'échelle microscopique, de la première micro-fissure, et ainsi de suite jusqu'à l'échelle du massif montagneux parcouru de failles.
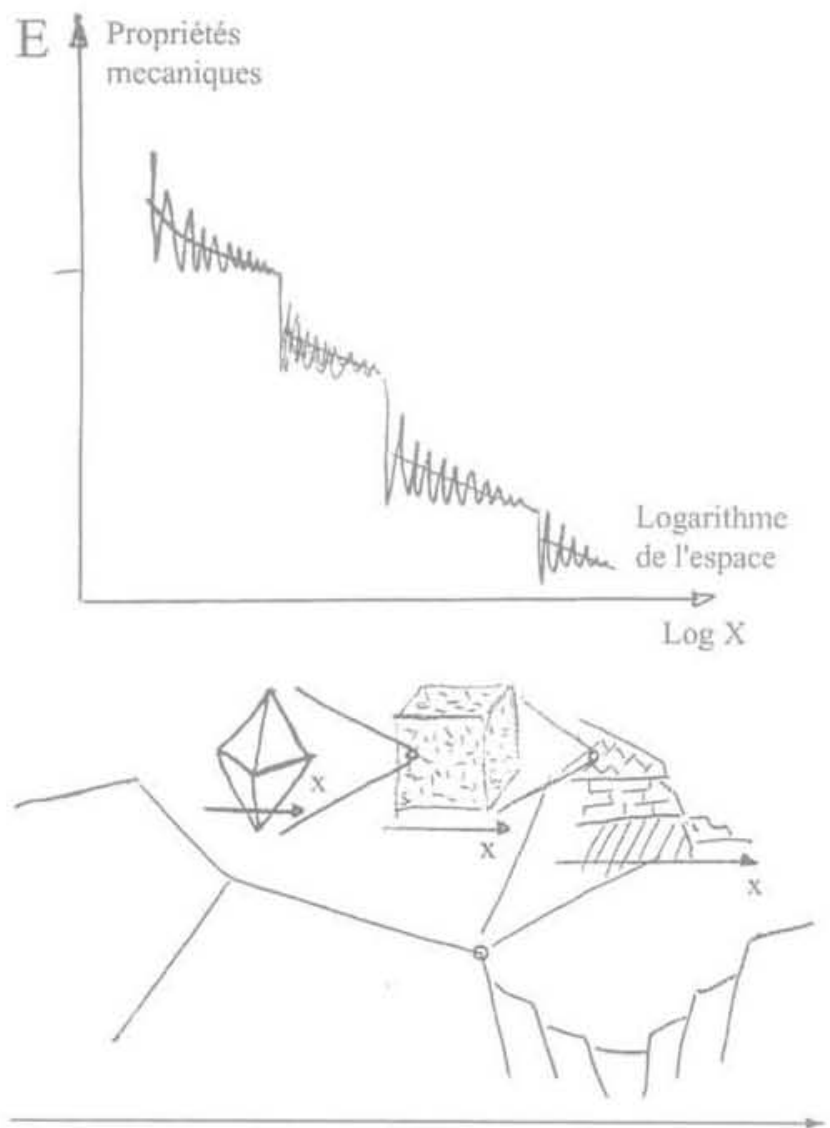

BG.1 Échelles emboitées pour les propriétés des roches.

Rock properties overlaped scales.

\section{2}

\section{Les trois types d'incertitudes}

\section{1,01}

\section{Incertitudes active et passive}

Mais ce désordre naturel n'est pas la seule incertitude dans la connaissance des propriétés des sols. Une deuxième source vient de notre connaissance imparfaite de la nature : la nature EST. Un phénomène physique incertain, quand il se réalise, se réalise de façon certaine ; il présente une valeur sûre, déterministe, mais nous ne pouvons pas la connaitre parfaitement à cause de l'imprécision et de l'imperfection de nos appareils de mesure. Nous commettons une erreur, distance entre une valeur vraie mais inconnue et une valeur donnée par l'appareil de mesure.

La première incertitude reliée à la réalisation du phénomène physique est souvent appelée active et la deuxième, liée à la mesure, est souvent appelée passive. 


\section{$1,2 \pi 2$}

\section{Erreur de modèle}

Enfin, il existe pour nous un troisième type d'incertitude, celle liée à l'utilisation de modèles de comportement. Les propriétés des sols et des roches rentrent dans des lois rhéologiques qui représentent plus ou moins bien la réalité de la relation contraintes-déformations. Ainsi on utilise essentiellement le modèle élastique linéaire isotrope-plastique parfait ou fragile, alors que le sol est mieux représenté par un modèle élastoplastique orthotrope. Quant au comportement réel du sol, personne ne le connaîtra jamais ; on ne fait que l'approcher. On fait donc une erreur de modèle indépendamment de l'erreur que l'on fait sur la mesure des paramètres du modèle choisi.

\section{2}

\section{Erreurs et incertitudes liées à la caractérisation des sols}

\section{1}

\section{Les erreurs d'observation et d'enquête}

\section{: 1 Th}

\section{La loi des erreurs}

La réalité est vraie, sûre mais inconnue. La mesure en est une estimation. On considérera donc la mesure comme une variable aléatoire. La différence entre la valeur vraie et la mesure est l'erreur de mesure $\varepsilon$ : c'est aussi une variable aléatoire.

Cette erreur peut avoir une part systématique : $E[\varepsilon] \neq 0$. On l'appelle un biais.

Elle a une part aléatoire $\sigma(\varepsilon) \neq 0$. C'est la dispersion de l'erreur ou incertitude.

La loi des erreurs est représentée par la fameuse courbe en cloche (Fig. 2). C'est la loi « normale » ou de Laplace-Gauss, appelée ainsi car elle représente le comportement des phénomènes incertains " normaux» qui dépendent d'un grand nombre de petites causes plus ou moins indépendantes et du même ordre de grandeur

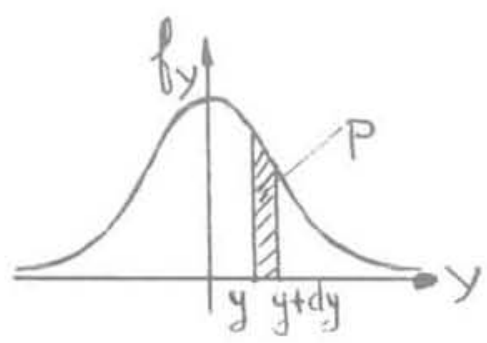

FG.2 Loi des erreurs.

Errors law.

\section{9tse}

\section{Erreurs d'observation}

On distingue trois types d'erreurs d'observation :

- Les erreurs de mesure proprement dites, $\varepsilon_{20}$ Elles sont liées à l'appareil de mesure et à l'opérateur.

Avec les progrès de la métrologie, on a considérablement réduit les erreurs d'imprécision et avec l'acquisition automatique, les erreurs d'opérateur. Encore faut-il avoir fait un étalonnage et un tarage corrects. Mais il reste les erreurs de reproductibilité liées au caractère destructif de la plupart des essais de géotechnique et les erreurs d'opérateur liées à la difficulté de certains essais (au triaxial, au pressiomètre : qualité du trou, etc.).

Enfin, il est bien connu que chaque essai représentant le " coup de main » de l'opèrateur, on constate des différences systématiques plus ou moins grandes d'un laboratoire ou d'un bureau de reconnaissance à l'autre.

\section{- Les erreurs de représentativité, $\varepsilon_{\text {rep }}$}

Elles proviennent de la transformation de la mesure physique.

On ne mesure quère directement que des longueurs, des masses et des températures et la plupart des appareils sont basés sur des mesures de déplacement. Il faut donc transformer la mesure. On procède alors à un étalonnage de l'appareil et à son tarage. Mais il y a des transformations plus importantes et plus incertaines : section d'un échantillon au triaxial pour la contrainte $\sigma_{1}$ ? Pente initiale d'une courbe de chargement pour le module de Young ? (en fait, il faut parler de modules sécants, la limite élastique sur chemin déviatorique ne dépassant guère $10^{-5}$ ).

- Les erreurs de l'instant, $\varepsilon_{\text {inst }}$

Elles proviennent de la variation de la propriété entre le moment où on la mesure et le moment où le sol est mis en cuvre.

Le cas typique est celui des propriétés du béton. Pour les sols, le remaniement relève de ce type d'erreur, en particulier pour tous les essais mécaniques de laboratoire et pour l'essai pressiométrique.

\section{D.1.7.}

\section{Les erreurs d'enquête}

\section{- Les erreurs d'enquête proprement dite}

Ce sont les erreurs liées à une mauvaise conduite des reconnaissances.

Les mesures effectuées ne sont pas représentatives du problème : on décrit en figure 3 l'exemple d'un remblai récent mal identifié au droit de chaque appui qui le sollicite, un remblai ancien en biseau pouvant exister sous certains appuis ; la figure 3 -b illustre le problème d'une fondation superficielle reconnue à moins de 3 à 5 fois sa largeur, ignorant ainsi une couche faible qui peut piloter les tassements.

\section{- Les erreurs d'échantillonnage}

Ce ne sont pas, à proprement parler, des erreurs. 

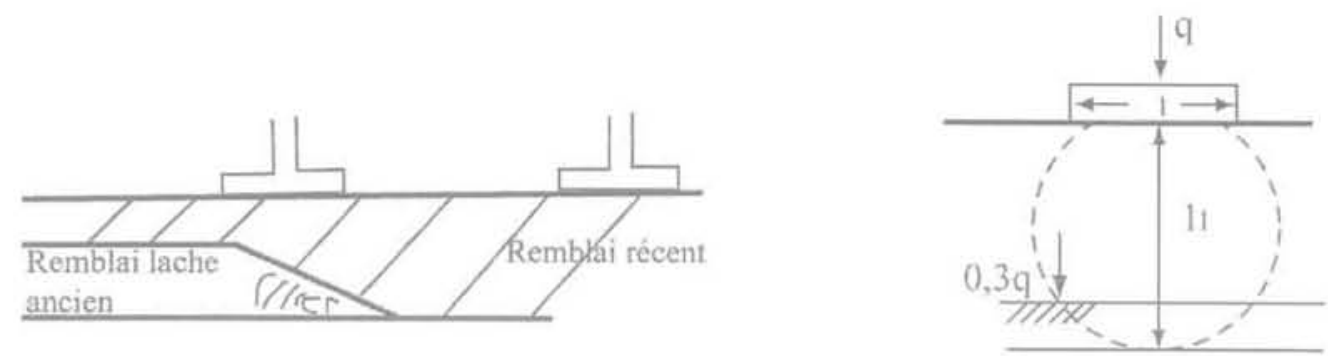

FIG.3 Exemples de problèmes d'enquête.

Investigation difficuities cases.

Imaginons que chaque mesure est parfaite mais que la propriété varie d'un point à l'autre de l'espace. Cette variabilité naturelle sera modélisée comme une variable aléatoire dont on va estimer les caractéristiques avec les mesures. On a ainsi un échantillon statistique de taille n pour estimer les caractéristiques d'une population infinie. On estime ces caractéristiques par des intervalles de confiance : la moyenne fou l'écart type) de la propriété, qui est sûre mais inconnue a une probabilité de $95 \%$ par exemple d'être encadrée par deux valeurs dépendant des observations. L'utilisation des intervalles de confiance est illustrée par les articles de Maurice Cassan "Utilisation de la statistique descriptive en géotechnique » et de François Baguelin et Jean-Bernard Kovaric « Une méthode de détermination des paramètres géotechniques ) pour le choix des paramètres de projet ou valeurs caractéristiques.

\section{Qins}

\section{Protection contre les erreurs d'observation et d'enquête et modélisation}

La modélisation des erreurs d'observation est naturellement faite par des variables aléatoires. On cherchera à définir en premier lieu l'espérance des erreurs $E[\varepsilon]$, les biais, et leur variance $\sigma^{2}(\varepsilon)$, leur dispersion. La protection contre ces différentes erreurs est claire puisque leurs causes sont claires : maitriser celles-ci. Une attention particulière doit être apportée à la réalisation des essais : réalisation des trous de sondages pour le prélèvement comme pour le pressiomètre, prélèvement et transport des carottes, conservation des carottes, découpage et mise en place des échantillons.

La modélisation des erreurs d'enquête proprement dite relève de la modélisation des erreurs humaines. Celle-ci n'est pas très avancée, spécialement en géotechnique. La seule parade est de ne jamais négliger le modèle géologique et de faire appel, pour numériser ce modèle, à un vrai géotechnicien.

Les erreurs d'échantillonnage relèvent de la théorie de l'estimation. Elles ne posent aucun problème de modélisation :

- l'article de Jean-Pierre Magnan « Quelques spécificités du problème des incertitudes en géotechnique » illustre le poids que peuvent revêtir celles de ces erreurs qui sont peu ou pas quantifiables ;

- l'article de Pierre Pouget et al. « Deux applications de l'analyse factorielle à des données géotechniques m montre tout le parti qu'on peut tirer des analyses factorielles lorsqu'on dispose d'un grand nombre de données, ici pour définir les modèles de terrain.

\section{2}

\section{La variabilité naturelle} sique.

C'est le caractère incertain du phénomène phy-

\section{mas?}

\section{La modélisation par des fonctions aléatoires de l'espace}

Les problèmes mécaniques sont des problèmes sur l'espace : une force est l'intégrale d'une contrainte sur une surface ou dans un massif, un déplacement est l'intégrale d'une déformation dans une direction ou dans un massif. Il s'agit donc d'intégrer des propriétés en tant que fonctions de l'espace. De plus, le sens commun veut qu'on juge les propriétés moyennes d'un volume de sol moins dispersées que ses propriétés ponctuelles : les fortes valeurs sont compensées par les faibles valeurs et plus le volume est grand plus le lissage est fort : on a bien plus de risque avec une semelle étroite qu'avec un radier.

On peut préciser cette idée avec l'exemple d'un essai au scissomètre de chantier :

Soit le profil de $\mathrm{Cu}$, en fonction de la profondeur en trait fin sur la figure 4 , enregistré tous les $10 \mathrm{~cm}$ avec des ailettes de $10 \mathrm{~cm}$. Si on avait fait l'enregistrement tous les $40 \mathrm{~cm}$ avec des ailettes de $40 \mathrm{~cm}$, on aurait obtenu la courbe en trait gras, dont chaque point est la moyenne de 4 points de la précédente. On obtient ainsi un profil moins dispersé. Ceci n'est ni plus ni moins que la règle de la variance de la moyenne de n valeurs, qui veut que celle-ci soit $n$ fois plus petite que la variance de chaque valeur:

Variance $[\bar{Z}]=$ Variance $[\mathrm{Z}] / n \quad$ avec $\bar{Z}=(1 / n) \Sigma[$

ou encore

$\sigma_{\overline{\mathrm{z}}}=\sigma_{\mathrm{z}} / \sqrt{\mathrm{n}}$ :

La dispersion du deuxième profil est deux fois plus petite que celle du premier profil. Cette question est facilement résolue dans le cas de valeurs discrètes indépendantes par la règle de la moyenne. Elle nécessite la définition de la fonction de covariance ou de la fonction d'auto corrélation des champs aléatoires et de la distance d'auto-corrélation a, distance à partir de laquelle les propriétés deviennent indépendantes, soit $1 \mathrm{~m}$ sur la figure 5 . On retrouve les mêmes notions 


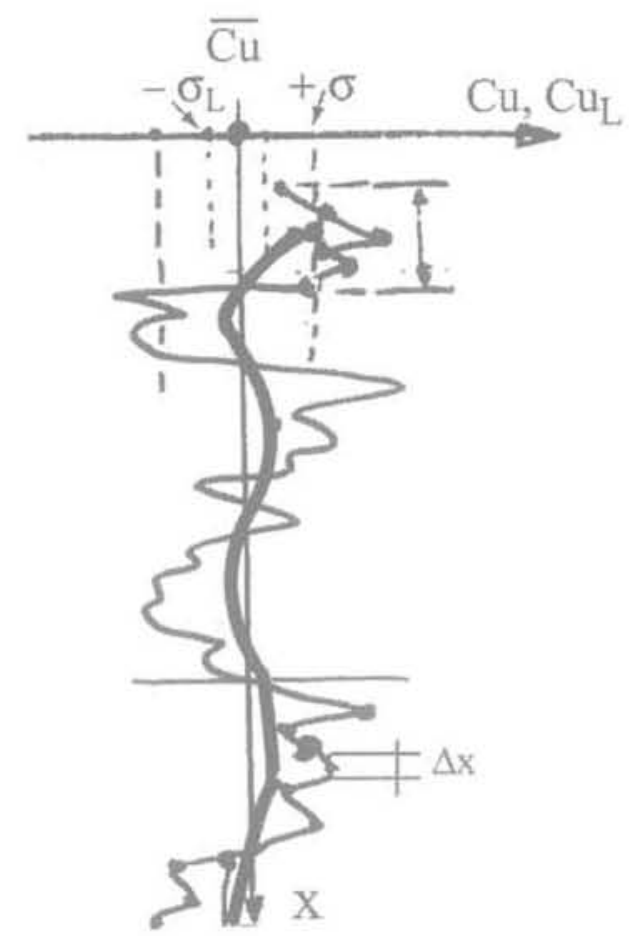

FG. 4 Profils scissométriques avec deux ailettes différentes.

Two different vane tests profiles.
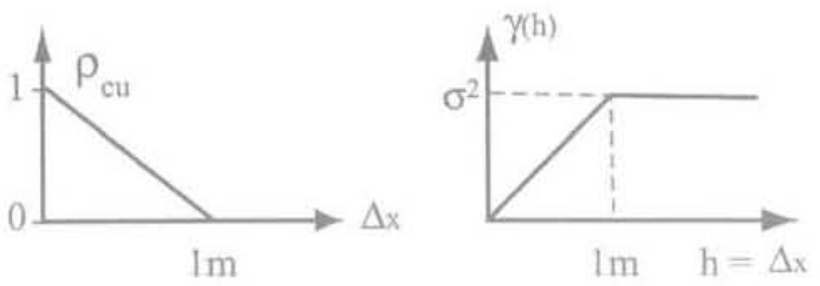

fig. 5 Corrélogramme et variogramme. Correlogram and variogram.

en géostatistique où le variogramme correspond au corrélogramme. Albert Bolle dans son article $\alpha$ étude et prise en compte de la variabilité naturelle » donne différents modèles pour la fonction d'auto corrélation et montre l'interprétation que l'on peut faire des corrélogrammes expérimentaux.

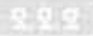

\section{Les premiers moments de la fonction aléatoire}

Ces premiers moments peuvent ètre illustrés par le profil d'un sondage pénétrométrique : à chaque niveau (x), tous les $2 \mathrm{~cm}$ par exemple, on enregistre une réalisation de la résistance de pointe $R p(x)$. On peut définir à chaque niveau les caractéristiques de la variable aléatoire « résistance de pointe ». Ces caractéristiques, l'espérance et la variance pour les deux premiers moments, deviennent des fonctions de l'espace $\bar{R} p(x)$ et $\sigma_{\text {Rp }}^{2}(x)$ (Fig. 6)

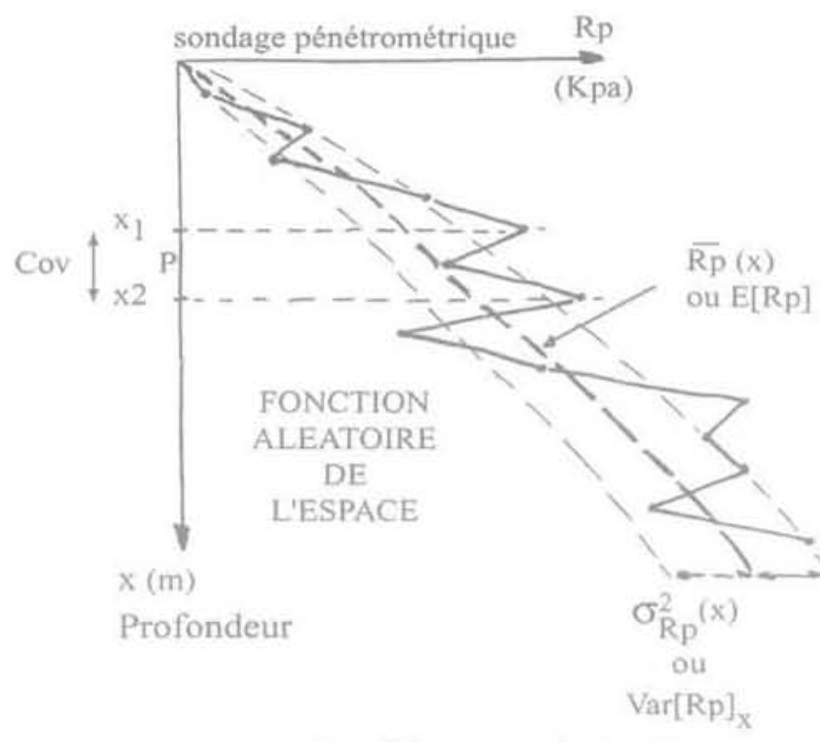

$\operatorname{Cov}\left[\operatorname{Rp}\left(x_{2}\right), \operatorname{Rp}\left(x_{1}\right)\right]$

FG.6 Résistance de pointe au pénétromètre comme fonction aléatoire de l'espace. Random field of cone penetration profile.

Pour être complet jusqu'à l'ordre 2, il faut définir la corrélation entre les différents niveaux. C'est la fonction d'auto covariance qui dépend des deux niveaux choisis $x_{1}$ et $x_{2}$ ou d'un des deux niveaux choisis $x$ et de l'écart entre lés deux niveaux $\Delta x$.

On cherchera, pour des commodités de modélisation et de traitement, à rendre le phénomène stationnaire, c'est-à-dire ayant les mêmes caractéristiques statistiques en tout point de l'espace. Cette exigence est très difficile à satisfaire et on se contentera de la stationnarité en moyenne d'ordre 2 qui rend seulement les deux premiers moments indépendants de l'espace.

Ainsi :

$\mathrm{E}\left[\mathrm{Rp}^{*}\right]=$ constante

$\operatorname{Var}\left[\mathrm{Rp}^{*}\right]=$ constante

Cov[Rp1* $\left.1^{*} \mathrm{Rp}^{*}\right]=\operatorname{Cov}[\Delta \mathrm{x}]$

$\mathrm{Rp}^{*}$ : transformée de Rp

\section{3}

\section{Prise en compte des erreurs, des incertitudes et de la variabilité naturelle}

\section{5 \\ Les erreurs d'observation}

Lorsqu'un paramètre physique entre dans une procédure de dimensionnement d'ouvrage, il devient un paramètre de calcul. Ce paramètre de calcul doit être unique, quel que soit le paramètre physique qui est à son origine : C'est par exemple la valeur naturelle $Z_{w}$. Mais on commet sur ce paramètre les erreurs d'observation déjà mentionnées:

- celles de l'appareil et de l'opérateur, e

- celles de représentation, $\mathrm{e}_{\text {rep' }}$. 
- celles de l'instant (ou remaniement pour la géotechnique), $\mathrm{e}_{\text {inst }}$

Ainsi, pour tout paramètre physique mesuré $\mathrm{Z}_{\mathrm{m}}$, on peut écrire :

$$
Z_{m}=Z_{i v}+e_{a a}+e_{\text {rep }}+e_{\text {inst }}
$$

$Z_{\mathrm{N}}$ étant la valeur naturelle ou vraie qui devient le paramètre de calcul

\section{- L'espérance ou le biais}

L'espérance de la valeur de calcul est alors :

$$
E\left[Z_{N}\right]=E\left[Z_{m}\right]-\left\{E\left[e_{a b}\right]+E\left[e_{\text {rop }}\right]+E\left[e_{\text {inst }}\right]\right\}
$$

On ne dispose pas de valeurs pour les biais qu'introduisent, sur la valeur mesurée par rapport à la valeur réelle, les différents facteurs (l'appareil, l'opérateur, les approximations dans la transformation de la mesure, le remaniement).

Le remaniement, la plupart du temps, minimise les valeurs de résistance du sol et semble être le biais le plus important en valeur absolue. Aussi en prenant :

$$
E\left[Z_{N}\right] \approx E\left[Z_{m}\right]
$$

on se place la plupart du temps du côté de la sécurité.

\section{- La dispersion}

La dispersion de la valeur de calcul est donnée par la variance :

$\operatorname{Var}\left[Z_{m}\right]=\operatorname{Var}\left[Z_{N}\right]+\operatorname{Var}\left[e_{\text {ao }}\right]+\operatorname{Var}\left[e_{\text {rep }}\right]+\operatorname{Var}\left[e_{\text {inst }}\right]$ $+\Sigma_{i=1 a 3} \operatorname{Cov}\left[Z_{N^{\prime}} e_{i}\right]+\Sigma_{i * 1}$ Cov $\left[e_{i}, e_{j}\right]$

On peut valablement supposer que les erreurs sont indépendantes des valeurs naturelles et qu'elles sont indépendantes entre elles (loi des erreurs), Alors $\operatorname{Cov}\left[Z_{N}, e_{i}\right]=\operatorname{Cov}[e, e]=0$.

Les variances étant des quantités positives, Var[e] $>$ 0 , alors :

$$
\operatorname{Var}\left[Z_{N}\right] \leq \operatorname{Var}\left[Z_{m}\right]
$$

Tout comme l'espérance, on ne connait pas la variance des différentes erreurs. Aussi, en prenant pour dispersion de la valeur de calcul celle des valeurs mesurées, on se place du côté de la sécurité.

\section{- La dépendance d'un point à l'autre de l'espace}

Elle est donnée par la covariance entre deux mesures distantes de $\Delta \mathrm{x}$ :

$$
\begin{gathered}
\operatorname{Cov}\left[Z_{m}(x), Z_{m}(x+\Delta x)\right]=\operatorname{Cov}\left[Z_{N}(x)+\Sigma_{1} e_{i}(x), Z_{N}(x+\Delta x)\right. \\
\left.+\Sigma_{i} e_{i}(x+\Delta x)\right]
\end{gathered}
$$

Les erreurs étant indépendantes entre elles (sauf pour $\Delta \mathrm{x}=0$ ) et indépendantes de la valeur à mesurer, on a, pour $\Delta x \neq 0$ :

$\operatorname{Cov}\left[Z_{m}(x), Z_{m}(x+\Delta x)\right] \approx \operatorname{Cov}\left[Z_{N}(x), Z_{N}(x+\Delta x)\right]$
A l'heure actuelle, en l'absence de modèles et de grandeurs pour les erreurs d'observation, on prend les valeurs mesurées ce qui, en général, place le géotechnicien du côté de la sécurité.

\subsection{9.}

\section{La variabilité naturelle et les erreurs d'échantillonnage}

\section{- Les incertitudes d'échantillonnage}

Les incertitudes d'échantillonnage, venant du nombre limité de mesures pour estimer $\mu=E\left[Z_{n}\right]$ et $\sigma=\sqrt{ } \operatorname{Var}\left[Z_{m}\right]$, sont prises en compte par des intervalles de confiance qui découlent de la théorie de l'estimation: On prend une forte probabilité $1-\alpha$ (en général $\alpha=5 \%$ ) pour que $\mu$ et $\sigma$ tombent dans un intervalle qui dépend du nombre $n$ de mesures faites et des valeurs de la moyenne empirique $m$ et de l'écart type estimé $S_{\text {. }}$ calculés avec ces mesures. Plus $n$ est faible, plus l'intervalle est grand, c'est-à-dire plus l'incertitude est grande. Les articles de Maurice Cassan et de François Baguelin et Jean-Bernard Kovaric utilisent largement cette théorie de l'estimation et des tests d'hypothèses.

\section{- La variabilité naturelle}

La variabilité naturelle $Z_{N}(x)$ est prise en compte par l'indice de sécurité $\beta$ qui permet théoriquement de calculer la probabilité de ruine. Le fait que cette résistance $Z_{\mathrm{N}}(\mathrm{x})$ soit intégrée dans un volume $\mathrm{V}$ concerné par le mécanisme (ou sur une surface $\Sigma$ ou une ligne L) est pris en compte par un champ de moyenne locale qui introduit une réduction de variance $x(\mathrm{~V})$. Cette réduction de variance est liée au coefficient d'auto corrélation de la manière suivante (à 1D) :

$$
\gamma(\mathrm{L})=2 / \mathrm{L} \cdot \int_{0}^{\mathrm{L}}(1-\Delta \mathrm{x} / \mathrm{L}) \cdot \mathrm{\rho}(\Delta \mathrm{x}) \cdot \mathrm{d}(\Delta \mathrm{x})
$$

Soit, avec le modèle triangulaire de corrélation de la figure 5 :

$$
\begin{aligned}
& \rho(\Delta)=|1-| \Delta x|/ a, \quad| \Delta x \mid \leq a \\
& 10 \text { sinon }
\end{aligned}
$$

$$
\begin{array}{rlr}
\gamma(\mathrm{L})=\mid 1-\mathrm{L} / 3 \mathrm{a} & \mathrm{L} \leq \mathrm{a} \\
\mid(\mathrm{a} / \mathrm{L})(1-\mathrm{a} / 3 \mathrm{~L}) & \mathrm{L} \geq \mathrm{a}
\end{array}
$$

Si le champ est séparable $(\gamma(\mathrm{V})=\gamma(\mathrm{L},) \cdot \gamma(\mathrm{L},) \cdot \gamma(\mathrm{L})$,$) , on$ peut connaître la réduction de variance à $3 \mathrm{D}$ de façon très simple en connaissant les distances d'auto corrélation a, $a_{y}$ et $a_{z}$ et les dimensions du volume sollicité $L_{x}$, $L_{y}$ et $L_{z}$.

Illustrons sur un exemple simple comment agit cette réduction de variance et comment on évalue la probabilité de ruine :

Soit une plate-forme offshore, par $100 \mathrm{~m}$ de fond, de poids déjaugé $\mathrm{P}^{\prime}=500000 \mathrm{t}$, à embase poids de $100 \mathrm{~m}$ de côté que l'on veut garantir, grâce à des bêches pénétrant de $\mathrm{D}=10 \mathrm{~m}$ dans le fond marin, contre le ripage 
vis-à-vis de forces horizontales (courants, houle, vent, etc.) extrêmes sur sa durée de vie (20 ans): $\bar{H}^{\prime}=200000$ $\mathrm{t}$ avec un écart type $\sigma_{\mathrm{H}}=20000 \mathrm{t}$. Les caractéristiques du fond marin, à $10 \mathrm{~m}$, sont (moyenne et coefficient de variation):

$$
\begin{aligned}
& \overline{\mathrm{c}^{\prime}}=28 \mathrm{t} / \mathrm{m}^{2} \quad \mathrm{CV}_{\mathrm{c}^{\prime}}=20 \% \text {, } \\
& \overline{\phi^{\prime}}=10^{\circ} \mathrm{CV},=13 \% \text {, avec CV }=\sigma_{x} \bar{x} \\
& \overline{\gamma^{\prime}}=10 \mathrm{KN} / \mathrm{m}^{3} \\
& \rho_{c o}=-0,6 \\
& \mathrm{CV}_{\gamma}=7 \% \\
& \operatorname{COV}\left(c^{\prime}, \phi^{\prime}\right)=p_{c o} \sigma_{c}, \sigma_{\phi} \text {. } \\
& a_{y}=a_{x}=33 \mathrm{~m}, \quad a_{2}=1,5 \mathrm{~m} \text { (les distances }
\end{aligned}
$$
d'auto-corrélation sont très différentes verticalement et horizontalement).

En faisant le calcul du coefficient de sécurité F avec les valeurs movennes, on obtient pour $R$ (résistance au cisaillement, uniquement sur la surface horizontale $\Sigma$ à $10 \mathrm{~m}$ sans compter les effets de bords) :

$$
\mathrm{R}=\Sigma \overline{\mathrm{C}^{\prime}}+\left(\mathrm{P}^{\prime}+\Sigma \overline{\gamma^{\prime} \mathrm{D}}\right) \operatorname{tg} \overline{\phi^{\prime}}=31,2.10^{4} \mathrm{t}
$$

$$
\begin{aligned}
& \mathrm{S}=\overline{\mathrm{H}}^{\prime}=20.10^{4} \mathrm{t} \\
& \text { d'où } \mathrm{F}=\mathrm{R} / \mathrm{S}=1,56
\end{aligned}
$$

Théoriquement, la probabilité de ruine est:

$p_{\mathrm{r}}=\operatorname{Prob}\left\{(R-S)=g(Z) \leq 0 \mid\right.$ avec $Z=\left\{c^{\prime}, \gamma, \operatorname{tg} \phi^{\prime}, H^{\prime}\right\rangle$

$\mathrm{R}-\mathrm{S}=\mathrm{g}(\mathrm{Z})$ est aussi appelé la fonction de performance ou marge de sécurité.

On montre (sous certaines conditions) qu'une valeur approchée de $\beta$ est :

$\beta=E[g(Z)] / \sigma_{g|z|}=(\bar{R}-\bar{S}) /\left(\sigma_{\mathrm{R}}^{2}+\sigma_{\mathrm{S}}{ }^{2}\right)^{1 / 2}$ (puisque R et $\mathrm{S}$ sont indépendants).

Une valeur approchée (sous certaines conditions) de p, est :

$$
p_{\mathrm{f}}=\Phi(-\beta)
$$

$\Phi$ étant la fonction de répartition de la loi normale.

En écrivant, par pure commodité, $\mathrm{Z}=\left|\mathrm{X}_{1}, \mathrm{X}_{2}, \mathrm{X}, \mathrm{X}_{4}\right|$ il vient, en divisant par la surface $\Sigma$ qui n'est pas incertaine:

$$
g(Z)=X_{4}+\left(a+b X_{2}\right) X_{3}-X_{4}^{\prime} \quad X_{4}^{\prime}=X_{4} / \Sigma
$$

On peut approximer $\mathrm{g}(\mathrm{Z})$ par un développement limité au premier ordre $g_{1}(Z)$ (linéarisation) en série de Taylor autour des valeurs moyennes:

$$
g_{1}(Z)=g(\bar{Z})+\Delta X_{1}+\left(a+b \bar{X}_{2}\right) \Delta X_{3}+b \bar{X}_{3} \Delta X_{2}-\Delta X_{4}^{\prime}
$$

On trouve que :

$$
E\left[g_{1}(Z)\right]=g(\bar{Z})=11,2 t / m^{2}
$$

et que :

$\operatorname{Var}\left[g_{1}(\mathrm{Z})\right]=\operatorname{Var}\left[\mathrm{X}_{1}\right]+\left(\mathrm{a}+\mathrm{b} \quad \overline{\mathrm{X}}_{2}\right)^{2} \operatorname{Var}\left[\mathrm{X}_{3}\right]+\left(\mathrm{b} \overline{\mathrm{X}}_{3}\right)^{2}$ $\operatorname{Var}\left[\mathrm{X}_{2}\right]+\operatorname{Var}\left[\mathrm{X}_{4}^{\prime}\right]+2\left(\mathrm{a}+\mathrm{bX} \bar{X}_{2}\right) \operatorname{COV}\left[\mathrm{X}_{1}, \mathrm{X}_{3}\right]=12,96+1,44+$ $0,02+4-5,2=13,2\left(\mathrm{t} / \mathrm{m}^{2}\right)^{2}$

soit $\sigma_{9112)}=3,6 \mathrm{t} / \mathrm{m}^{2}$

Alors, on trouve que $\beta=E\left[g_{1}(Z)\right] / s_{\text {n1tz }}=3,1$, ce qui conduit à une probabilité de ruine $\mathrm{p}_{\mathrm{f}}=10^{3}$ inacceptable.

Nous venons de dire qu'il ne faut pas prendre, pour les variances, la valeur ponctuelle mais la valeur réduite: $\operatorname{Var}\left[\mathrm{X}_{\mathrm{v}}\right]=\operatorname{Var}[\mathrm{X}], \gamma(\mathrm{V}), \mathrm{X}_{\mathrm{v}}$, étant le champ de moyenne locale sur $\mathrm{V}$.

La réduction de variance pour ' $c^{\prime}, \operatorname{tg} \phi^{\prime}$ et $\gamma$ est alors $\Delta(\Sigma)=\gamma(\mathrm{L})^{2}$ :

avec $\gamma(\mathrm{L})=(33 / 100)(1-33 / 300)$ (on $\mathrm{a} \mathrm{L}=100 \mathrm{~m}$; $\mathrm{a}=$ $33 \mathrm{~m})$

$(\gamma$ serait aussi à intégrer suivant la verticale sur la fenêtre D)

$$
\gamma(\Sigma)=0,086
$$

En appliquant cette réduction aux variances et covariances de $c^{\prime}, \operatorname{tg} \phi^{\prime}$ et $\gamma$ (mais pas à $H^{\prime}$ ) la variance de $g_{3}(Z)$ devient :

$$
\operatorname{Var}\left[g_{1}(Z)\right]=1,11+0,12+0,00+4-0,45
$$

soit $\quad \operatorname{Var}[R]=0,78$ (au lieu de 9,22)

et $\operatorname{Var}[S]=4$ (inchangé)

On trouve $\beta_{1}=5,11$, soit une probabilité de ruine de l'ordre de $210^{-7}$.

Il est intéressant de voir l'évolution de la contribution respective de la résistance et de la sollicitation (équations 17) ainsi que la contribution de chaque variable $\mathrm{X}$, d'une part par sa dispersion propre (valeurs 8) mais encore par le gradient $\delta g(Z) / \delta X$ (équations 14 et 16). P. Londe distinguait ainsi les paramètres "lourds".

Des méthodes similaires ont été développées lorsque l'êtat limite n'est pas donné par uné fonction explicite mais par un calcul aux éléments finis ; c'est ce qu'on appelle la méthode des éléments finis stochastiques qui est présentée dans l'article de Gabriel Auvinet et al, \& La méthode des éléments finis stochastiques en géotechnique. y Enfin, Albert Bolle propose dans son article « Étude et prise en compte de la variabilité spatiale n) une nouvelle technique de sirnulation fort peu coûteuse en temps calcul et qui évite de passer par les approximations des développements en série de Taylor.

Ces méthodes sont encore loin d'être courantes dans l'ingénierie qui utilise plutôt des méthodes dites « semi-probabilistes » consistant à retenir une valeur caractéristique tirée de l'observation statistique et de procéder à un calcul déterministe avec des coefticients pondèrateurs pour tenir compte de différentes sources d'incertitudes supplémentaires.

L'article de Maurice Cassan donne un exemple de choix de valeur caractéristique, utilisant l'analyse sta- 
tistique pour la variabilité naturelle et l'échantillonnage et utilisant l'expérience pour la réduction de variance tandis que celui de François Baguelin et Jean-Bernard Kovaric intègre explicitement cette dernière à partir de la dimension de l'ouvrage et de la distance d'auto corrélation. Cette procédure a été proposée pour l'Eurocode 7.

\section{3}

\section{Erreurs de modèle de comportement et autres erreurs}

Nous ne parlons pas explicitement de l'erreur de modèle au cours de ces exposés, Cette notion est très claire théoriquement mais très imprécise pratiquement. Son traitement peut être inclus dans celui des erreurs mal définies ou imprécises grâce aux « sous-ensembles flous $»$ : Daniel Boissier, dans son article « Décision et incomplétude o donne divers exemples d'utilisation des « sous-ensembles flous » pour caractériser les imprécisions sur les paramètres de dimensionnement. Cette procédure peut être explicitement étendue aux biais apportés par les modèles de calcul. On peut en effet montrer (Favre et Touati, 1995) que l'erreur de modèle est un biais pur sans dispersion mais il est impossible de l'isoler complètement de l'erreur d'observation sur ses paramètres.

Parmi les autres erreurs, il y a les approximations d'intégration (calculs aux éléments finis, etc.). De façon plus générale, j’ai présenté au colloque « Risque et Génie civil ») (Favre, 2000) l'ensemble des aléas dans les ouvrages géotechniques et leur importance relative, la nature, l'identification et la modélisation de ceux liés au terrain.

\section{4}

\section{Poids respectifs \\ de la variabilité naturelle et de l'échantillonnage}

On vient de voir que la variabilité naturelle était prise en compte par l'indice de sécurité $\beta$ calculé à partir des champs de moyenne locale et non à partir des paramètres aléatoires « ponctuels ». Par contre l'incertitude sur les moments (espérance, variance et auto covariance) de ces paramètres est prise en compte avec des intervalles de confiance. Un exemple de leur prise en compte conjointe est donné par l'article de François Baguelin et Jean-Bernard Kovaric mais sans qu'apparaisse leur poids respectif sur la probabilité de ruine de l'ouvrage.

Cette question nous parait suffisamment importante pour qu'on puisse attirer l'attention sur elle à partir d'un exemple académique (Favre, 1988).

Considérons que toutes les variables de base entrant dans la fonction de performance $g(Z)$ ont fait l'objet du mème échantillonnage de taille $n$.

On cherche une borne inférieure ${ }^{\text {II }}$ à $\beta$ au seuil $\alpha$. c'est-à-dire une valeur $\beta_{0}$ telle que :

$$
\operatorname{Prob}\left(\beta \leq \beta_{0}\right) \leq a
$$

(1) Plus $\beta$ est petit, plus la probabilité de ruine est grande.

On vient de voir (\$ 2.3.2) qu'une forme approchée de l'indice de sécurité $\beta$ est :

$\beta=E[g(Z)] / \sigma_{\text {grzp }}$ où $p_{t}=\Phi(-\beta)$ est la probabilité de ruine.

On cherche la borne $\beta_{0}$ sous la forme $\mathrm{m}_{\min } / \mathrm{s}_{\max }$ Soit $m_{\min }-m_{\operatorname{mix}}$ l'intervalle de confiance de $E[g(Z)]$ au seuil $\alpha$

$$
\begin{gathered}
\operatorname{Prob}\left\{m_{\min } \leq E[g(Z)] \leq m_{\text {max }}\right]=1-\alpha \\
\operatorname{Prob}\left(E\left[g(Z) \leq m_{\text {min }}\right]\right)=\alpha / 2=P(A)
\end{gathered}
$$

Soit $s_{\min }-s_{\max }$ l'intervalle de confiance de $\sigma_{g(z)}$ au seuil $\alpha$

$$
\begin{gathered}
\text { Probls } \left._{\min } \leq \sigma_{g(z)} \leq \mathrm{s}_{\max }\right)=1-\alpha \\
\text { Prob }\left(\mathrm{s}_{\max } \leq \sigma_{g(z)}\right)=\alpha_{2}=\mathrm{P}(\mathrm{B})
\end{gathered}
$$

Les valeurs de $m_{\min }$ et $\mathrm{s}_{\max }$ sont données dans l'article de Maurice Cassan.

La probabilité pour que $\beta=E[g(Z)] / \sigma_{\text {olz }}$ soit inférieur à $\beta_{0}=m_{\min } / s_{\text {max }}$ est la probabilité de trouver le point de coordonnées $\{\mathrm{E}[\mathrm{g}(\mathrm{Z})]$; $\sigma \mathrm{g}(\mathrm{Z})\}$ sous la droite $E[g(Z)]=\sigma_{\text {otzl }} \cdot\left(m_{\min } / s_{\max }\right)($ Fig. $7-a)$, soit dans le domaine $\bar{D}$. Ce domaine est plus petit que le domaine $\bar{D}$ * (Fig. 7-b).

Le domaine $\bar{D}^{*}$ est l'union des domaines $A$ et $B$ moins leur intersection, soit :

$\operatorname{Prob}(\beta \in D) \leq \operatorname{Prob}\left(\beta \in D^{*}\right)=P(A)+P(B)-P(A \cap B) \leq \alpha$ car $P(A \cap B)$ est positif comme toute mesure de probabilité.

Ainsi,

Prob $\left(\beta \leq \beta_{0}\right) \leq \alpha \quad$ avec $p_{0}=\Phi\left(-\beta_{0}\right)$

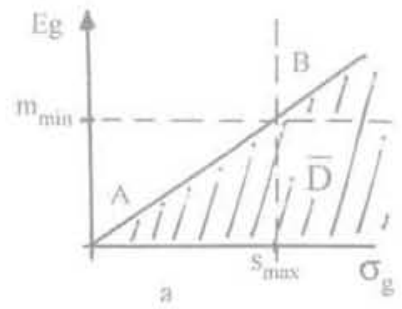

FG, 7 Domaine de dépassement du seuil $\beta_{0}$ (a) et domaine majorant (b).

Thereshold $\beta_{0}$ exceeding domain (a) and raising of this one (b).

Comparons maintenant le poids respectif de la variabilité naturelle et des incertitudes de mesures (cf. tableau ci-après).

Soit par exemple un mur poids sur semelle filante de $2 \mathrm{~m}$ de large et $10 \mathrm{~m}$ de long. On considère que la charge appliquée au sol est constante et connue (cas favorable). Dans ce cas l'indice de sécurité peut s'écrire 
TABLEAU Rôle conjugué de la dispersion naturelle et de la reconnaissance. Natural variability and investigation joint effects.

\begin{tabular}{|c|c|c|c|}
\hline \multicolumn{2}{|l|}{$\operatorname{cv}(\mathrm{Z})$} & $20 \%$ & $40 \%$ \\
\hline \multicolumn{2}{|c|}{$\mathrm{cv}(\mathrm{R})=\sqrt{ } \gamma \mathrm{cv}(\mathrm{Z})$} & $5 \%$ & $10 \%$ \\
\hline \multirow[t]{2}{*}{$\mathrm{n}=\infty}$, & \multirow[t]{5}{*}{$\mathrm{CV}(\mathrm{R})=\mathrm{CV}(\mathrm{R})$} & $5 \%$ & $10 \%$ \\
\hline & & $\mathrm{F}=3, \beta_{0}=13,3$ & $F=3, \beta_{0}=6,7$ \\
\hline \multirow{3}{*}{$p_{r}=p_{0}$} & & $\mathrm{p}_{0}=0$ & $p_{0}=0$ \\
\hline & & $F=2, \beta_{0}=10$ & $\mathrm{~F}=2, \beta_{0}=5$ \\
\hline & & $\mathrm{p}_{0}=0$ & $P_{0} \leq 10^{-7}$ \\
\hline \multirow[t]{2}{*}{$n=8$} & \multirow[t]{2}{*}{$\mathrm{CV}(\mathrm{R}) \max =$} & $8 \%$ & $17 \%$ \\
\hline & & $\mathrm{F}=3, \beta_{0}=8,2$ & $\mathrm{~F}=3, \beta_{0}=3,92$ \\
\hline \multirow{3}{*}{\multicolumn{2}{|c|}{$\begin{array}{l}\text { Il y a moins de } 2 \text { chances } \\
\text { sur } 10 \text { d'avoir } \\
\mathrm{p}_{1} \geq \mathrm{p}_{0}\end{array}$}} & $\mathrm{P}_{0}=0$ & $\mathrm{P}_{0}=5.10^{-5}$ \\
\hline & & $\mathrm{F}=2, \mathrm{~B}_{0}=6,2$ & $F=2, \beta_{0}=2,94$ \\
\hline & & $p_{0}=0$ & $p_{0}=1,6 \cdot 10^{-3}$ \\
\hline \multirow[t]{2}{*}{$\mathrm{n}=4$} & \multirow[t]{2}{*}{$\mathrm{CV}(\mathrm{R})_{\max }=$} & $12 \%$ & $26 \%$ \\
\hline & & $F=3, \beta_{0}=5,5$ & $F=3, \beta_{0}=2,56$ \\
\hline \multirow{3}{*}{\multicolumn{2}{|c|}{$\begin{array}{l}\text { Il y a moins de } 2 \text { chances } \\
\text { sur } 10 \text { d'avoir } \\
p_{f} \geq p_{0}\end{array}$}} & $p_{0} \leq 10^{-8}$ & $p_{0}=5.10^{-3}$ \\
\hline & & $\mathrm{F}=2, \beta_{0}=4,2$ & $F=2, \beta_{0}=1,92$ \\
\hline & & $\mathrm{p}_{0}=10^{-6}$ & $\mathrm{P}_{0}=2,8.10^{-2}$ \\
\hline
\end{tabular}

simplement en fonction du coefficient de sécurité $F$ et du coefficient de variation de la capacité portante ultime $\mathrm{cv}(\mathrm{R})$ :

$$
\beta=(1-1 / \mathrm{F}) / \operatorname{cv}(\mathrm{R})
$$

Le sol est soit assez homogène (avec un coefficient de variation des propriétés cv(Z) de l'ordre de $20 \%$ ), soit peu homogène (cv(Z) de l'ordre de $40 \%$ ) (ligne 1 du tableau I)

On fait l'hypothèse que la réduction de variance est importante (cas favorable) et vaut $1 / 16$; cela divise le coefficient de variation pour la capacité portante ultime $\mathrm{cv}(\mathrm{R})$ par 4 (ligne 2).

a) On suppose qu'on connait parfaitement de sol, ce qui correspond à un échantillonnage infini (lignes 3).

Alors cv(R) est parfaitement déterminé : son estimateur $\mathrm{CV}(\mathrm{R})$, variable aléatoire, est égal à cv(R) (ligne 31). Alors $\beta=\beta_{0}$ et $p_{t}=p_{0}$.

Le calcul donne, pour un coefficient de sécurité normal $\mathrm{F}=3$ (ligne 3-2), un indice de sécurité très grand, que le sol soit homogène ou non, correspondant à des probabilités de ruine très faibles. Pour un coefficient de sécurité faible $\mathrm{F}=2$ (ligne $3-3$ ), il donne un indice de sécurité moyen dans le cas du sol hétérogène et la probabilité de ruine commence à ne pas être négligeable de $10^{-0}$ à $10^{-}$. Ceci signifierait que dans le cas d'un sol hétérogène, même si on le connaît très bien, on prend un petit risque avec un coefficient de sécurité faible.

b) Supposons maintenant qu'on connait imparfaitement le sol. On a fait 8 mesures (lignes 4).
On ne peut estimer avec certitude le coefficient de variation de la capacité portante. Soit CV(R) la borne supérieure de cv(R) telle que :

$$
\text { Problev }(\mathrm{R}) \geq \mathrm{CV}(\mathrm{R})_{\text {max }} \mid=20 \%
$$

avec $\beta_{0}=(1-1 / F) / C V\left(R_{\max }\right)$

Pour le sol assez homogène, la borne passe à $8 \%$. Pour le sol hétérogène, elle passe à $17 \%$ (ligne 4-1).

Pour le sol homogêne, la valeur de $\beta_{0}$ reste forte et la probabilité de ruine est non significative. Par contre, pour le sol hétérogène, les valeurs deviennent faibles et les probabilités $p_{0}$ deviennent significatives. Mais la probabilité de ruine reste acceptable car on n'a que 2 chances sur 10 d'avoir une probabilité de ruine $p_{\text {f }}$ inférieure à $\mathrm{p}_{0}$. On pourrait l'apprécier en écrivant:

$$
\mathrm{p}_{\mathrm{r}}=2 \cdot 10^{-1} \cdot \mathrm{p}_{0}
$$

Ainsi la probabilité de ruine serait de $10^{-5}$ avec un coefficient de sécurité normal (ligne 4-2) et de $310^{-4}$ avec un coefficient faible ce qui est presque excessif (ligne 4-3).

c) Enfin, supposons qu'on connaisse mal le sol. On n'a fait que quatre mesures (lignes 5). La borne $C V(R)_{\text {max }}$ devient très grande (ligne $5-1$ ). Pour le sol homogéne, que le coefficient de sécurité soit normal ou faible, le risque est très faible. Par contre, pour le sol hétérogène, même avec un coefficient de sécurité normal (ligne 5-2), il est inacceptable (une fondation sur mille aurait des désordres).

Cet exemple n'est qu'illustratif ; il ne donne en aucune façon des valeurs absolues de probabilités de 
ruine. Il montre seulement comment le risque évolue en fonction des différents aléas :

- la variabilité naturelle, à elle seule semble ne pas peser (lignes 3). Rappelons tout de même que nous nous sommes mis dans un cas avantageux avec une forte réduction de la variance et une action non aléatoire. Si cette réduction n'avait été que de 1/4, les $\beta$ auraient doublés et on voit toutes les conséquences sur les $p_{0}$ dans les 3 cas (lignes 3,4 et 5 ) ;

- on voit l'effet très pénalisant du manque de reconnaissance dès que le sol n'est pas assez homogène. On ne saurait qu'insister sur la nécessité de faire des reconnaissances assez conséquentes dès que le sol est hétérogène :

- enfin, on retrouve la légitimitè de prendre des coefficients de sécurité faibles dans des circonstances favorables (sol assez homogène et bonne reconnaissance).

\section{Conclusion}

Nous venons de dégager trois grands types d'incertitudes pour l'examen des propriétés des sols, qui tiennent à leur variabilité naturelle, à leurs connaissances et aux modèles que l'on choisit pour ces propriétés.

Les incertitudes de modèle sont des biais, inaccessibles pour le moment sauf par une procédure indirecte basée sur le retour d'expérience d'ouvrages réels (Favre, 1998). L'article de J.-P. Magnan montre l'importance de certains de ces aspects peu quantifiables.

Les articles de M. Cassan et de F. Baguelin et J.B. Kovaric traitent tout d'abord de l'exploitation des mesures et de leur modélisation. Le but recherché à travers la modélisation est de réduire au maximum l'incertitude sur les paramètres de calcul des ouvrages, que cette incertitude vienne de la variabilité naturelle, de l'échantillonnage ou du lissage par intégration (réduction de variance). Ils indiquent les techniques classiques de modélisation statistique des paramètres fonction ou non de la profondeur et les techniques de tests statistiques. De plus, au cas oủ l'ingénieur disposerait de la mesure de plusieurs paramètres un nombre non négligeable de fois, on peut utiliser les techniques d'analyses factorielles (Favre, 1983) comme le montre l'article de P. Pouget et al.

L'article d'A. Bolle indique comment modéliser les paramètres non plus en fonction de la simple profondeur mais en fonction de tout l'espace par des champs aléatoires. Il dégage la notion d'auto corrélation qui introduit celle de réduction de variance, absolument fondamentale dans les calculs de probabilité de ruine des ouvrages en terre. Il donne une nouvelle méthode de simulation pour ce type de calcul.

G. Auvinet et al. traitent ensuite de la prise en compte de cette incertitude dans le dimensionnement des ouvrages en exposant la théorie de la fiabilité et son application au dimensionnement par la méthode des élements finis stachastiques. Comme nous venons de le préciser, il s'agit de la prise en compte de la variabilité naturelle puisque nous sommes amenés à supposer que $Z_{m} \approx Z_{N}$. Ils ne prennent pas en compte les erreurs de modèles vu qu'à l'heure actuelle les propositions dans ce sens commencent à peine à apparaître (Favre et al., 1998). De mon côté, j'ai indiqué plus haut comment juger du poids respectif de la variabilité naturelle et des incertitudes d'échantillonnage.

Enfin, D. Boissier considère l'application possible en matière de prise de décision lorsque les données sont imprécises et incertaines au point de ne pouvoir faire l'objet d'un traitement statistique. Il montre le parti qu'on peut tirer des sous-ensembles flous.

\section{Bibliographie}

Favre J.-L., (1983) - a Analyses statistiques comparées de pénétromètres. scissomètres et pressiomètres auto-foreurs 10 . Symp. Int. Reco. Sols Roches par essais en place, Paris. Int Assoc Eng Geology, vol.2, p. 287-29?

Favre J.-L. (1988) - Special Lecture « Properties of soil : large uncertainties - a minor aspect of foundation reliability $n$. Symposium on Reliability-Based Design in Civil Engineering. Lausanne, Swiss
Federal Institute of Technology, vol. 2. p. $51-66$.

Favre J,-L., Touati K. (1995) - a Probabilistic estimation of the errors in finite element calculation code for shallow foundation 3. Proc, 7th Int. Conf. Applic. Stat. Prob. soils struct. eng.. Paris, A. A. Bakema; vol. 2. p. 1309-1315.

Favre J.-1., Biarez J., Hachi F. (1998) - w An only model for sand and clay p. PROBA. MAT 21st Century, PERM. NATO-ASI
Series, Kluwer academic publishers, vol. $46, p, 465-481$

Favre J.-L. (1998) - " Errors in geotechnics and their impact on safety 1. . Int. Conf, on Uncertain Structures, Miami 1996. Computers and Structures, 67. p. $37-45$.

Favre J.-L. (2000) - $\alpha$ Les incertitudes géologiques et qéotechniques is. Risque et Génie civil, Paris, Presses des Ponts et Chaussées, p. 187-229. 